\title{
Can immune biomarkers predict benefit from targeted agents in metastatic renal cell carcinoma?
}

\author{
Renate Pichler ${ }^{1}$, Manuela Schmidinger ${ }^{2}$ \\ ${ }^{1}$ Department of Urology, Medical University of Innsbruck, Innsbruck, Austria; ${ }^{2}$ Department of Medicine I, Clinical Division of Oncology and \\ Comprehensive Cancer Center, Medical University of Vienna, Vienna, Austria \\ Correspondence to: Renate Pichler, MD, PhD. Department of Urology, Medical University Innsbruck, Anichstraße 35, A-6020 Innsbruck, Austria. \\ Email: renate.pichler@i-med.ac.at; Manuela Schmidinger, MD. Department of Medicine I, Clinical Division of Oncology and Comprehensive \\ Cancer Center, Medical University of Vienna, Waehringer Guertel 18-20, A-1090 Vienna, Austria. Email: Manuela.schmidinger@meduniwien.ac.at. \\ Provenance: This is an invited article commissioned by the Section Editor Dr. Xiao Li (Department of Urology, Jiangsu Cancer Hospital \& Jiangsu \\ Institute of Cancer Research \& Affiliated Cancer Hospital of Nanjing Medical University, Nanjing, China). \\ Comment on: Flaifel A, Xie W, Braun DA, et al. PD-L1 Expression and Clinical Outcomes to Cabozantinib, Everolimus, and Sunitinib in Patients \\ with Metastatic Renal Cell Carcinoma: Analysis of the Randomized Clinical Trials METEOR and CABOSUN. Clin Cancer Res 2019;25:6080-8.
}

Submitted Oct 14, 2019. Accepted for publication Nov 28, 2019.

doi: 10.21037/atm.2019.11.138

View this article at: http://dx.doi.org/10.21037/atm.2019.11.138

We read with great interest the article by Flaifel and colleagues (1), who interrogated the predictive and prognostic value of (I) PD-L1 expression [on tumor and immune cells (IC)], (II) the extent of immune cell infiltrates and (III) the cMET expression in patients receiving targeted agents (TA). The authors analyzed pre-treatment tumor tissue obtained from patients from two randomized trials, the METEOR trial (2) (cabozantinib versus everolimus in $2^{\text {nd }}$-line) and the CABOSUN trial (3) (cabozantinib versus sunitinib in $1^{\text {st }}$-line). The main findings were first, that PD-L1 expression on tumor cells (TC) is associated with poor clinical outcome in all patients and independently of the TA received and second, that cabozantinib is superior to everolimus and sunitinib in terms of PFS and OS, irrespective of PD-L1 expression.

Research on predictive biomarkers is of paramount importance, since treatment decisions in metastatic renal cell carcinoma (mRCC) have become increasingly difficult. A plethora of agents has been established in the last decade. In 2019, thirteen different systemic treatment strategies exist (4). Results from more recently conducted randomized phase III trials have established both cabozantinib and immune check point inhibitor combinations [nivolumab + ipilimumab (5), pembrolizumab + axitinib (6), avelumab + axitinib (7)] as the new standard of care in $1^{\text {st }}$-line mRCC. Finally, as all of these agents have been compared to sunitinib, the role of other established $1^{\text {st }}$-line antiangiogenic drugs such as tivozanib (8) remains unclear.

The scenario physicians currently face in mRCC is an abundance of treatment options that were shown to be superior to sunitinib, but no answer to the question: which treatment for which patient? Although some of these strategies have been investigated in specific subgroups [intermediate and poor IMDC risk group for nivolumab + ipilimumab (5) and cabozantinib (3)], the population for which treatment decisions need to be made is quite large. No phase III trials have directly compared novel agents yet. The effort to initiate and conduct such comparative phase III studies is subject to several hurdles: first, new agents are not developed simultaneously, which makes a prompt comparison of new compounds difficult; second, the choice of the comparator depends on the time when the study is initiated; third, study designs are biased by the sponsor, who seeks to establish a new compound rapidly in a crowded market. Various biomarker studies such as the one by Flaifel and colleagues (1) try to find answers to questions that should have been addressed in clinical trials.

The present work may be useful for physicians in clinical practice. The results are not meant to outline the precise therapeutic strategy for the individual patient; they rather help to identify the first therapeutic direction via analysis of PD-L1 expression on TC. 
Table 1 Ongoing clinical trials combining cabozantinib and checkpoint inhibitors in mRCC frontline treatment

\begin{tabular}{llccccc}
\hline Study name & Targeting agents & Comparison & Phase & Primary endpoint & Status* & NCT number \\
\hline CheckMate 9ER & Cabozantinib + nivolumab & Sunitinib & III & PFS & Active, not recruiting & NCT03141177 \\
COSMIC-313 & Cabozantinib + nivolumab + ipilimumab & $/$ & III & PFS & Recruiting & NCT03937219 \\
NA & Cabozantinib + pembrolizumab & / & I/II & ORR & Recruiting & NCT03149822 \\
NA & Cabozantinib + avelumab & / & I & Safety, tolerability & Recruiting & NCT03200587 \\
\hline
\end{tabular}

*, status according to https://clinicaltrials.gov/, accessed on 03 October 2019. NA, not assessed; ORR, objective response rate; PFS, progression-free survival.

Interrogation of the predictive value of PD-L1 expression has led to controversial results in the past; in the CheckMate214 trial (5), patients with PD-L1 positive tumors were found to benefit most from the immune check point inhibitor combination, with unprecedentedly high rates of complete remission (5). However, the role of PDL1 expression was less clear in immune checkpoint inhibitor (ICPI)-tyrosine kinase inhibitor (TKI) trials $(6,7)$. Finally, in $2^{\text {nd }}$-line setting, PD-L1 expression was not found to be predictive for nivolumab (9).

The work by Flaifel and colleagues (1) is the first to address the role of PD-L1 expression in patients receiving TA. TA were shown to possess immune-modulatory properties, leading to an immune permissive tumor microenvironment $(10,11)$. Taking this into consideration, together with the challenge of choosing among various strategies, it seems appropriate to investigate as to whether PD-L1 might be predictive for TA. According to the report of Flaifel and colleagues (1) cabozantinib seems to be more effective in PD-L1 positive patients when compared to sunitinib or everolimus especially in progression-free survival (PFS); thus, if immune-check point inhibitors are not available or contraindicated for any reason, cabozantinib appears to be the treatment of choice. In addition, the authors found cabozantinib to be more effective than sunitinib and everolimus in the PD-L1 negative setting. The authors draw the conclusion that a cabozantinib-based treatment should be offered to PD-L1 negative or PD-L1 unselected patients. Based on their dataset, this appears to be a very reasonable approach.

However, the data should not lead to the assumption that cabozantinib is the only option in PD-L1 unclear and PD-L1-negative patients. It needs to be highlighted that this research was restricted to patients from studies where only sunitinib and everolimus were the comparators. We cannot extrapolate from the current findings that cabozantinib is superior to other TKIs such as axitinib, tivozanib, lenvatinib, which also were found to have antiinflammatory and immunomodulatory features $(12,13)$. Nevertheless, cabozantinib might be particularly effective, since it inhibits not only VEGFR2, AXL and RET, but also the c-MET-signaling. The c-MET axis might be a critical driver of a neutrophil-mediated reactive resistance program to cancer immunotherapy. In detail, c-MET signaling is responsible for mobilizing a subset of (c-MET+) neutrophils from the bone marrow into a $\mathrm{T}$ cell-inflamed microenvironment during immunotherapy in vivo. This reactive recruitment of neutrophils limits the efficacy of immunotherapy due to acquired immunosuppressive properties by suppressing therapy-induced $\mathrm{T}$ cell expansion and effector functions (14-16). This observation may explain why Flaifel and colleagues (1) found a positive correlation between MET and PD-L1 expression. Thus, concomitant c-MET inhibition may indeed enhance the efficacy of immunotherapy by impairing the reactive mobilization of neutrophils into tumors and lymph nodes (14). The potential of cabozantinib as an attractive targeted candidate for combination-based therapies in the first-line setting is now being tested in clinical trials as summarized in Table 1.

The authors should be commended for their efforts to obtain precise and reliable data on PD-L1 expression. PDL1 expression was evaluated on TC and IC, confirming a positive PD-L1 status ( $\geq 1 \%$ cutoff) on TC in $23 \%$ (CABOSUN) and 29\% (METEOR), and on IC in 59\% (METEOR) and 61\% (CABOSUN), respectively. This is comparable to the results from CheckMate 214 (TC PD-L1: 24\%), KEYNOTE-426 (IC PD-L1: 60\%) and JAVELIN Renal 101 (IC PD-L1: 63\%) studies, although different PD-L1 immunohistochemistry assays were used (5-7). Thus, the PD-L1 antibody used in this trial (405.9A11) showed excellent concordance with the other commercially available PD-L1 antibodies (CheckMate 214: 28-8; KEYNOTE-426: 22C3; JAVELIN Renal 101: SP263) 
(5-7). Moreover, to overcome inter-observer variability and heterogeneity when evaluating PD-L1 expression, the authors developed a double PD-L1 assay to simultaneously quantify PD-L1 on both TC and IC, providing congruent data with published results.

What remains as a limitation of the study is that all investigations were primarily performed from archival tumor tissue, mostly from the primary tumor. This offers only a snapshot on the individual renal cancer biology, given the well-known heterogeneity of PD-L1 expression within the tumor microenvironment, the biological variability of immune cell infiltration both within primary tumors and among RCC metastases $(17,18)$.

In this context, the findings by Flaifel and colleagues (1) raise relevant clinical questions: if PD-L1 expression can, as shown here, help to make treatment decisions, it appears increasingly important to perform biopsies from spatially separated metastases prior to starting systemic therapy. Such an approach may be justified in routine clinical practice. A more precise biological picture of the disease in each individual patient is urgently needed to spare the patient unsuccessful treatment lines. It also may help to reduce the financial burden of expensive therapeutic combinations with improved cost-effectiveness.

In conclusion, the authors could elegantly demonstrate that the presence or absence of PD-L1 expression on TC may help to choose between cabozantinib, sunitinib and everolimus. The reliability of such information could be improved by using fresh tumor tissue, against which the future treatment is directed. The authors should be encouraged to expand their work to RCC patients receiving either dual immune check point blockade or combinations of ICPI with various different TKIs.

\section{Acknowledgments}

None.

\section{Footnote}

Conflicts of Interest: R Pichler: Honoraria for lectures and advisory boards: Pfizer, BMS, Roche, Ipsen, MSD, Merck, EISAI. Travel grants: BMS, Pfizer, Roche, Pierre Fabre. Research grants: Astellas, Agea Pharma. M Schmidinger: Honoraria for lectures and advisory boards: Pfizer, BMS, Novartis, Roche, Ipsen, Exelixis, EISAI, EUSA, Stellas. Research Grants: Roche, Pfizer. Travel grants: Roche, Ipsen, Pfizer.
Ethical Statement: The authors are accountable for all aspects of the work in ensuring that questions related to the accuracy or integrity of any part of the work are appropriately investigated and resolved.

\section{References}

1. Flaifel A, Xie W, Braun DA, et al. PD-L1 Expression and Clinical Outcomes to Cabozantinib, Everolimus, and Sunitinib in Patients with Metastatic Renal Cell Carcinoma: Analysis of the Randomized Clinical Trials METEOR and CABOSUN. Clin Cancer Res 2019;25:6080-8.

2. Choueiri TK, Escudier B, Powles T, et al. Cabozantinib versus everolimus in advanced renal cell carcinoma (METEOR): final results from a randomised, open-label, phase 3 trial. Lancet Oncol 2016;17:917-27.

3. Choueiri TK, Halabi S, Sanford BL, et al. Cabozantinib Versus Sunitinib As Initial Targeted Therapy for Patients With Metastatic Renal Cell Carcinoma of Poor or Intermediate Risk: The Alliance A031203 CABOSUN Trial. J Clin Oncol 2017;35:591-7.

4. Lalani AA, McGregor BA, Albiges L, et al. Systemic Treatment of Metastatic Clear Cell Renal Cell Carcinoma in 2018: Current Paradigms, Use of Immunotherapy, and Future Directions. Eur Urol 2019;75:100-10.

5. Motzer RJ, Tannir NM, McDermott DF, et al. Nivolumab plus Ipilimumab versus Sunitinib in Advanced Renal-Cell Carcinoma. N Engl J Med 2018;378:1277-90.

6. Rini BI, Plimack ER, Stus V, et al. Pembrolizumab plus Axitinib versus Sunitinib for Advanced Renal-Cell Carcinoma. N Engl J Med 2019;380:1116-27.

7. Motzer RJ, Penkov K, Haanen J, et al. Avelumab plus Axitinib versus Sunitinib for Advanced Renal-Cell Carcinoma. N Engl J Med 2019;380:1103-15.

8. Motzer RJ, Nosov D, Eisen T, et al. Tivozanib versus sorafenib as initial targeted therapy for patients with metastatic renal cell carcinoma: results from a phase III trial. J Clin Oncol 2013;31:3791-9.

9. Motzer RJ, Escudier B, McDermott DF, et al. Nivolumab versus Everolimus in Advanced Renal-Cell Carcinoma. N Engl J Med 2015;373:1803-13.

10. Sharma P, Allison JP. The future of immune checkpoint therapy. Science 2015;348:56-61.

11. Liu XD, Hoang A, Zhou L, et al. Resistance to Antiangiogenic Therapy Is Associated with an Immunosuppressive Tumor Microenvironment in Metastatic Renal Cell Carcinoma. Cancer Immunol Res 
2015;3:1017-29.

12. Marinelli Busilacchi E, Costantini A, Viola N, et al. Immunomodulatory Effects of Tyrosine Kinase Inhibitor In Vitro and In Vivo Study. Biol Blood Marrow Transplant 2018;24:267-75.

13. Santoni M, Berardi R, Amantini C, et al. Role of natural and adaptive immunity in renal cell carcinoma response to VEGFR-TKIs and mTOR inhibitor. Int J Cancer 2014;134:2772-7.

14. Glodde N, Bald T, van den Boorn-Konijnenberg D, et al. Reactive Neutrophil Responses Dependent on the Receptor Tyrosine Kinase c-MET Limit Cancer Immunotherapy. Immunity 2017;47:789-802.e9.

15. Ciamporcero E, Miles KM, Adelaiye R, et al. Combination strategy targeting VEGF and HGF/c-met in human renal cell carcinoma models. Mol Cancer Ther 2015;14:101-10.

16. Nandagopal L, Sonpavde GP, Agarwal N. Investigational MET inhibitors to treat Renal cell carcinoma. Expert Opin Investig Drugs 2019;28:851-60.

17. Baine MK, Turcu G, Zito CR, et al. Characterization of tumor infiltrating lymphocytes in paired primary and metastatic renal cell carcinoma specimens. Oncotarget 2015;6:24990-5002.

18. Bersanelli M, Gnetti L, Varotti E, et al. Immune context characterization and heterogeneity in primary tumors and pulmonary metastases from renal cell carcinoma. Immunotherapy 2019;11:21-35.
Cite this article as: Pichler R, Schmidinger M. Can immune biomarkers predict benefit from targeted agents in metastatic renal cell carcinoma? Ann Transl Med 2019;7(Suppl 8):S275. doi: 10.21037/atm.2019.11.138 